\title{
Analyse der Wechselwirkungen statischer Körperteilhaltungen mittels physiologischer Erfassung der Beanspruchung
}

\author{
Tobias Hellig ${ }^{1}$ Laura Johnen ${ }^{1}$ Alexander Mertens ${ }^{1} \cdot$ Verena Nitsch $^{1} \cdot$ Christopher Brandl $^{1}$ \\ Online publiziert: 15 . Oktober 2018 \\ ○ Der/die Autor(en) 2018
}

\section{Zusammenfassung}

Die Beurteilung der Belastung durch Körperhaltung erfolgt derzeit vielfach ohne Berücksichtigung von Wechselwirkungen einzelner Körperteilhaltungen. In diesem Beitrag werden Ergebnisse einer empirischen Laborstudie vorgestellt, welche eine systematische Analyse der Wechselwirkungen von Körperteilhaltungen des Rückens und der Schulter auf die Muskelaktivität von acht Muskeln mittels Oberflächenelektromyographie und auf das Beanspruchungsempfinden beinhaltet. An dieser Studie nahmen 50 Versuchspersonen teil. Hierzu wurden jeweils vier Stufen der Ventralneigung des Rumpfes (Schrittweite von $20^{\circ}$ ) und der Flexion der Schulter (Schrittweite von $30^{\circ}$ ) untersucht. Eine inferenzstatistische Auswertung der Ergebnisse mittels einer multivariaten Varianzanalyse mit Messwiederholung zeigte sowohl signifikante Haupteffekte der Ventralneigung und der Schulterflexion als auch signifikante Interaktionseffekte der untersuchten Variablen auf sechs Muskeln und das Beanspruchungsempfinden. Die Berücksichtigung von Wechselwirkungen bei der Bewertung von Körperhaltungen kann dazu beitragen, Muskel- und Skeletterkrankungen in Zukunft wirksamer zu begegnen.

Praktische Relevanz. Der Beitrag stellt Auswirkungen auf die muskuloskelettale Beanspruchung des menschlichen Körpers bei der Kombination von Rumpfneigung und Schulterflexion vor, deren Berücksichtigung bei der Belastungsbewertung einen wichtigen Beitrag zur Vermeidung schädlicher Körperhaltungen liefert und so zu einer Reduzierung von Muskelund Skeletterkrankungen beitragen kann.

Schlüsselwörter Ergonomie $\cdot$ Körperhaltung $\cdot$ Wechselwirkungen $\cdot$ Belastungsanalyse

Dipl.-Ing. Tobias Hellig

t.hellig@iaw.rwth-aachen.de

1 Lehrstuhl und Institut für Arbeitswissenschaft, RWTH

Aachen, Bergdriesch 27, 52062 Aachen, Deutschland 


\title{
Investigation of interaction effects of static working postures based on objective measurement of exposure variables
}

\begin{abstract}
Today, the assessment of working postures is based on postural load of individual body segments with disregard to interaction effects of working postures. This study introduces results of an empirical laboratory study investigating interaction effects of the back and the shoulder on muscle activity of eight muscles based on surface electromyography and rating of perceived exertion. 50 participants volunteered in this study. Four levels of back flexion (factor interval of $20^{\circ}$ ) and shoulder flexion (factor interval of $30^{\circ}$ ) were taken as independent variables. Repeated-measures MANOVA revealed significant effects of back flexion, shoulder flexion, and its interaction on muscle activity of six muscles, and rating of perceived exertion. Thus, the consideration of interaction effects of working postures in assessments of musculoskeletal exertion can contribute towards an effective prevention of musculoskeletal disorders.

Practical Relevance. This paper presents effects of musculoskeletal workload on the human body during a combined back flexion and shoulder flexion. Consideration of interaction effects in exposure assessment improves the avoidance of hazardous working postures and contributes to the reduction of musculoskeletal disorders.
\end{abstract}

Keywords Ergonomics - Working postures - Interaction effects - Exposure assessment

\section{Einleitung}

Die europäische Norm DIN EN 1005-4 (2009) sowie Brandl et al. (2017b) und Widanarko et al. (2012) beschreiben einen Zusammenhang zwischen ungünstigen Körperhaltungen und dem damit einhergehenden Risiko der Entstehung von Muskel- und Skeletterkrankungen. Diese sind in Deutschland sowie weltweit führende Ursache von Schmerzen und Funktionseinschränkungen (Robert Koch-Institut 2015). Grundlage ergonomischer Interventionsprozesse zur Verringerung von Muskel- und Skeletterkrankungen und Verbesserung von Arbeitssystemen durch eine Reduktion von Belastungen bildet die Analyse und Bewertung der vorherrschenden Belastungen im Arbeitssystem. Hierzu steht eine große Anzahl an Methoden zur Verfügung, welche von Denis et al. (2000), David (2005), Takala et al. (2010) sowie Brandl (2017) übersichtlich zusammengefasst und beschrieben werden. Eine Analyse und Bewertung von Körperhaltungen erfolgt hauptsächlich unter Anwendung von beobachtungsbasierten Methoden, wie z.B. dem Ovako Working Posture Analysing System (OWAS) (Karhu et al. 1981) oder dem Rapid Upper-Limb Assessment (RULA) (McAtamney und Corlett 1993). Solche beobachtungsbasierten Belastungsbewertungsverfahren beurteilen Körperhaltung auf Basis eines relativ vereinfachten Körpermodells, welches bspw. Körperteilhaltungen der oberen Extremitäten, des Rückens und der unteren Extremitäten erfasst, klassifiziert und anschließend eine summarische Gesamtbewertung der Körperhaltung unter Berücksichtigung der einzelnen Körperteilhaltungen ermöglicht (Roman-Liu 2014). Bezüglich der Gesamtbewertung von Körperhaltung bestehen jedoch aufgrund des gegenwärtigen Kenntnisstandes Einschränkungen hinsichtlich der Berücksichtigung von Wechselwirkungen der einzelnen Körperteilhaltungen
(DIN EN 1005-4 2009; Lim et al. 2011). Grundlage für die Gesamtbewertung von Körperhaltung darf nicht allein die Bewertung der einzelnen Körperteilhaltungen sein, vielmehr ist eine Berücksichtigung von Wechselwirkungen zwischen den einzelnen Körperteilen notwendig, da aus einer Kombination von akzeptablen Körperteilhaltungen auch nicht akzeptable Körperhaltungen entstehen können (DIN EN 1005-4 2009). Neben beobachtenden Verfahren zur Bewertung von Körperhaltung stehen auch objektive Messverfahren sowie Verfahren zur Erfassung des Beanspruchungsempfindens zur Verfügung (Schlick et al. 2018). Durch eine Erfassung geeigneter physiologischer oder biochemischer Messgrößen am Menschen ist eine induktive Ermittlung der Beanspruchung (Rohmert 1983) aufgrund statischer Körperhaltungen und deren Risikobeurteilung möglich. Aufgrund mangelnder Praktikabilität stellt die objektive Beanspruchungsermittlung keine geeignete Vorgehensweise für die betriebliche Praxis dar. Jedoch ist auf Basis der Ergebnisse solcher objektiver Beanspruchungsermittlungen eine Risikobeurteilung von Körperhaltungen unter Einbeziehung von Wechselwirkungen zwischen Körperteilhaltungen durch erweiterte beobachtungsbasierte Verfahren möglich.

Wechselwirkungen von Körperteilhaltungen sind Betrachtungsgegenstand verschiedener empirischer Untersuchungen. Plewa et al. (2016) weisen signifikante Wechselwirkungen zwischen der Unterarm- und Handhaltung auf das maximale Drehmoment des Handgelenks nach. In einer Untersuchung von Wechselwirkungen von Unterarmund Handgelenkhaltung auf das Beanspruchungsempfinden beschreiben Khan et al. (2009) signifikante Wechselwirkungen der Unterarmhaltung und des Kraftniveaus auf das Beanspruchungsempfinden. Farooq und Khan (2014) beschreiben Wechselwirkungen zwischen Schulterrotation 
und Ellenbogenflexion auf das Beanspruchungsempfinden und die Muskelaktivität des Extensor carpi radialis brevis (kurzer radialer Handstrecker). Neben zahlreichen Studien, welche Wechselwirkungen des Schulter-Hand-Armsystems untersuchen, sind Wechselwirkungen zwischen Körperteilhaltungen des Rückens und weiteren Körperteilhaltungen lediglich Betrachtungsgegenstand in der Studie von Lim et al. (2011), welche signifikante Wechselwirkungen zwischen der Ventralneigung des Rumpfes und der Schulterflexion auf die Muskelaktivität von Muskeln des Oberkörpers und des Armes sowie auf das Beanspruchungsempfinden zeigen. Gleichwohl nimmt insbesondere der Rücken eine zentrale Stellung für die Entstehung von Muskel- und Skeletterkrankungen ein (Luger et al. 2015; Widanarko et al. 2011). Die in den genannten Studien ermittelten Wechselwirkungen zwischen Körperteilhaltungen markieren weiteren Forschungsbedarf zur systematischen und vollständigen Analyse von Wechselwirkungen.

Ziel dieses Beitrags ist die systematische Untersuchung von Wechselwirkungen des Rückens und des Armes mittels Oberflächenelektromyographie (OEMG) und der Erhebung der subjektiven Beanspruchung. Für die statistische Untersuchung der Forschungsfrage wird angenommen, dass mit einer zunehmenden Ventralneigung des Rumpfes sowie mit einer zunehmenden Schulterflexion die Beanspruchung ansteigt. Zudem wird angenommen, dass Ventralneigung des Rumpfes und Schulterflexion einen wechselseitigen Einfluss auf die Beanspruchung ausüben.

\section{Methode}

\subsection{Beschreibung der Stichprobe}

An der Studie zur Analyse der Wechselwirkungen von statischen Körperteilhaltungen nahmen 50 Versuchsteilnehmer (29 Männer, 21 Frauen) im Alter von 20 bis 47 Jahren $(\mathrm{M}=25,48 \mathrm{a} ; \mathrm{SD}=4,01 \mathrm{a})$ teil. Aufgrund von muskuloskelettalen Beschwerden wurden drei Versuchsteilnehmer von der Datenauswertung ausgeschlossen. Auf die Auswahl einer nach Geschlecht ausbalancierten Stichprobe wurde verzichtet, da geschlechtsspezifische Unterschiede in der normalisierten Muskelaktivität nicht zu erwarten sind (Brandl 2017; Srinivasan et al. 2016).

Jeder Versuchsteilnehmer wurde über mögliche Risiken wie Reizung der Haut durch die Hautvorbereitung zur Elektrodenapplikation aufgeklärt. Muskuloskelettale Beschwerden der Versuchsteilnehmer, welche im Zeitraum von sechs Monaten vor der Studienteilnahme auftraten, wurden als Ausschlusskriterium definiert und vor Studienbeginn durch einen Fragebogen erhoben.

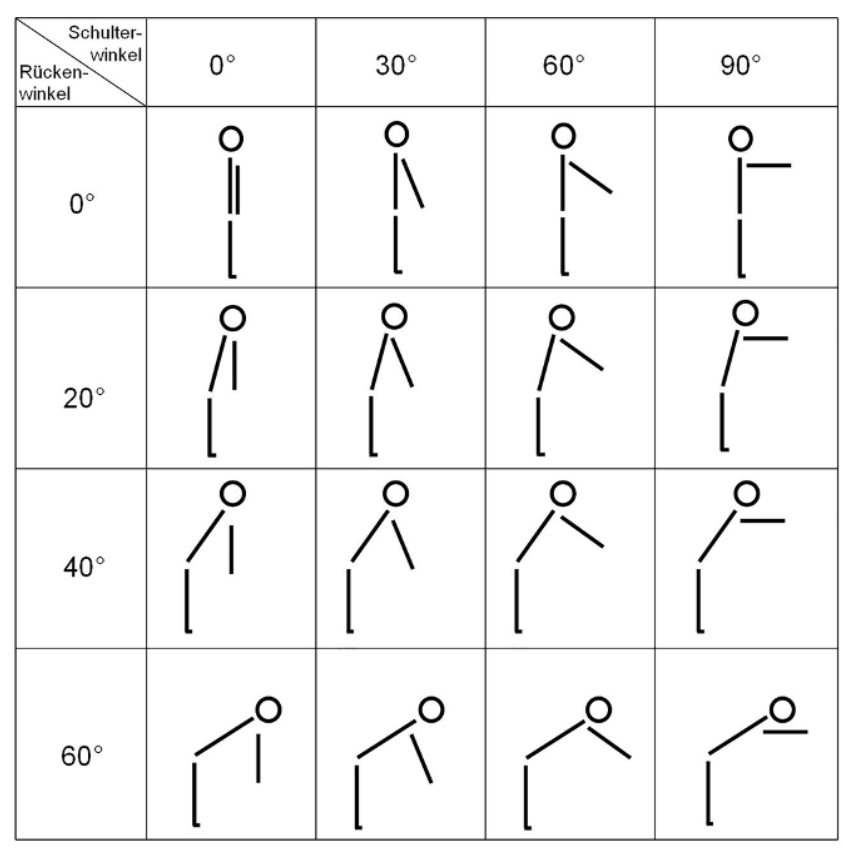

Abb. 1 Untersuchte statische Körperhaltungen

Fig. 1 Different types of investigated working postures

\subsection{Studiendesign}

Im Rahmen der Studie nahmen die Versuchsteilnehmer 16 verschiedene statische Körperhaltungen für die Dauer von jeweils einer Minute ein. Die untersuchten Körperhaltungen sind in Abb. 1 dargestellt. Eine Körperhaltung setzte sich aus der Kombination einer Ventralneigung des Rumpfes und einer Flexion der Schulter in stehender Haltung zusammen. Der Ventralneigungswinkel wurde mit einer Schrittweite von $20^{\circ}$ variiert, sodass Ventralneigungen von $0^{\circ}, 20^{\circ}, 40^{\circ}$ sowie $60^{\circ}$ (B0, B20, B40 und B60) untersucht wurden. Der Ventralneigungswinkel des Rumpfes entsprach dabei dem Winkel zwischen der senkrechten Längsachse des Rumpfes und der Senkrechten zum Boden. Der Schulterflexionswinkel wurde in einer Schrittweite von $30^{\circ}$ variiert, sodass Schulterflexionen im Winkel von $0^{\circ}, 30^{\circ}, 60^{\circ}$ und $90^{\circ}$ (S0, S30, S60 und S90) untersucht wurden. Für jede Ventralneigung entsprach ein Flexionswinkel der Schulter von $0^{\circ}$ einer Armhaltung senkrecht zum Boden. Die weiteren Flexionswinkel der Schulter entsprachen dem Winkel zwischen der Senkrechten zum Boden und der Längsachse des ausgestreckten Armes. Folglich bedeutete eine Schulterflexion von $90^{\circ}$ für jeden Ventralneigungswinkel eine horizontal nach vorne gerichtete Armhaltung. Die Variationsbreite der Körperwinkel wurde gewählt, um einen möglichst großen Bereich der praxisrelevanten Ventralneigung des Rumpfes und der Schulterflexion bei gleichzeitig akzeptabler Studiendauer zu untersuchen. 
Während der isometrischen Kontraktionsphase wurde die elektrische Muskelaktivität der folgenden acht Muskeln (vier Muskelpaaren) im Bereich der Lendenwirbelsäule, der Schulter und des oberen Rückens mittels OEMG erfasst: Linker und rechter Musculus (M.) trapezius pars descendens (LUT, RUT), linker und rechter M. trapezius pars ascendens (LLT, RLT), linker und rechter M. anterior deltoideus (LAD, RAD) und linker und rechter M. erector spinae (LES, RES). Die Auswahl der untersuchten Muskeln erfolgte auf Basis der durch die Auslenkung des Rumpfes und der Schulter beanspruchten Muskeln. Um den Einfluss von Muskelermüdung auf die Ergebnisse der Messungen zu eliminieren, erfolgte eine Randomisierung der Reihenfolge der Faktorstufenkombinationen.

Zudem wurde im Anschluss an jede isometrische Kontraktionsphase der 16 Körperhaltungen das Beanspruchungsempfinden für den ganzen Körper anhand der CR10-Skala nach Borg (1990) erfasst. Während der Abfrage des Beanspruchungsempfindens war die Skala mit den beschreibenden Worten für die Versuchsteilnehmer sichtbar platziert, sodass diese nicht memoriert werden musste.

Zusätzlich hielten die Versuchsteilnehmer während der isometrischen Kontraktionsphase in jeder Hand ein Gewicht von $0,5 \mathrm{~kg}$. Im Anschluss an jede isometrische Kontraktionsphase wurde eine Pause von jeweils einer Minute Dauer eingehalten. Nach jeweils vier isometrischen Kontraktionsphasen (alle Kombinationen der verschiedenen Schulterflexion mit einer Ventralneigung des Rumpfes) hielten die Versuchsteilnehmer eine Pause von fünf Minuten ein.

\subsection{Vorgehensweise}

Die Vorgehensweise im Rahmen dieser Studie bestand aus zwei Phasen: (1) Hautvorbereitung und Elektrodenapplikation sowie (2) isometrische Kontraktionsphase der statischen Körperhaltungen.

Die Hautvorbereitung sowie die Elektroden- und Sensorapplikation wurden in Übereinstimmung mit den diesbezüglichen Empfehlungen der Europäischen Richtlinien für OEMG (SENIAM) (Hermens 1999) durchgeführt. Hierbei wurden die Haare der betreffenden Körperpartien entfernt und mittels einer abrasiven Paste die Hautoberfläche gereinigt. Die Applikation der Oberflächenelektroden erfolgte auf dem gemäß den standardisierten Funktionstests zur Positionsbestimmung nach Perotto und Delagi (2011) palpierten Muskelbauch. Die Oberflächenelektroden wurden in Längsrichtung des Muskels aufgeklebt und der OEMGSensor parallel zum Muskel angebracht. Zur Stabilisierung des elektrischen Zustands wurde nach der Applikation der Oberflächenelektroden eine Wartezeit von fünf Minuten eingehalten. Zudem wurde die Signalqualität vor Beginn der Aufzeichnung durch eine visuelle Kontrolle sichergestellt (Iridiastadi und Nussbaum 2006). Hieran schloss sich die Erfassung der elektrischen Muskelaktivität während einer maximalen willkürlichen isometrischen Muskelkontraktion (MVC) zur Normalisierung der unter den Versuchsbedingungen erfassten elektrischen Muskelaktivität an.

In der zweiten Phase wurden die beschriebenen 16 statischen Körperhaltungen eingenommen und gehalten. Die Einhaltung der vorgegebenen Körperhaltung durch die Versuchsperson über die Dauer der isometrischen Kontraktionsphase von jeweils einer Minute wurde durch eine studienspezifische Software unter Nutzung der Microsoft Kinect V2 sichergestellt (Brandl et al. 2016). Den Startzeitpunkt der messtechnischen Erfassung der Muskelaktivität markierte jeweils die korrekte Einnahme der geforderten Körperhaltung.

\subsection{Datenerfassung und Datenaufbereitung}

Im Rahmen dieser Studie wurden Silber/Silberchloridbasierte Einmalelektroden mit adhäsiv-Gel (Abmessungen: $4 \times 2,2 \mathrm{~cm}$; Durchmesser der aktiven Leitfläche: $1 \mathrm{~cm}$; Zwischenelektrodenabstand: $1,75 \mathrm{~cm}$ ) verwendet und entsprechend der SENIAM-Richtlinien platziert (Hermens 1999). Das EMG-Signal wurde um den Faktor 1000 verstärkt, die Input-Impedanz des Verstärkers betrug $100 \mathrm{M} \Omega$, die Gleichtaktunterdrückung $100 \mathrm{~dB}$. Die Erfassung des EMG-Signals erfolgte mit einer Abtastrate von $1500 \mathrm{~Hz}$ in einem Frequenzbereich von 10 bis $500 \mathrm{~Hz}$ (Bandpass). Die Muskelaktivitäten wurden mittels einer kabellosen Messeinrichtung (Desktop DTS Receiver, Noraxon, USA) aufgenommen und mittels der Software MyoMuscle v3.8 (Noraxon, USA) visualisiert, nachbearbeitet und gespeichert. Zur Signalnachbearbeitung wurden eine EKG-Unterdrückung (Kluth et al. 2013) und eine Glättung der Messkurve durch den RMS-Algorithmus mit einem Fenster von $100 \mathrm{~ms}$ (Hermens 1999) angewandt. Bei den Amplituden der 16 Körperhaltungen fanden eine Amplitudennormalisierung zum Peak-Wert der jeweiligen maximalen Willkürkontraktion und eine Reskalierung auf die prozentuale Maximalkontraktionskraft (\% MVC) statt (Hermens 1999; Sousa und Tavares 2012).

\subsection{Datenauswertung}

Um die Effekte der Rumpfneigung und Schulterflexion (unabhängige Variablen) auf die normierte elektrische Muskelaktivität (sEA) der acht Muskeln und auf das Beanspruchungsempfinden (abhängige Variablen) zu untersuchen, wurde eine multivariate Varianzanalyse mit Messwiederholung (MANOVA) auf einem Signifikanzniveau für den Fehler 1. Art von $\alpha=5 \%$ durchgeführt. Entsprechend der Empfehlung von Field (2013) wurde zum Report der multivariaten Tests die Pillai-Spur verwendet. Um die Effekte der 
Tab. 1 Ergebnisse der separaten ANOVAs für Effekte der Rumpfneigung, Schulterflexion sowie deren Interaktion auf sEA und Beanspruchungsempfinden

Table 1 Results of seperate ANOVAs for effects of back inklination, shoulder flexion and its interaction on muscle activity and rating of perceived exertion

\begin{tabular}{|c|c|c|c|c|c|c|c|}
\hline Unabhängige Variable & $\begin{array}{l}\text { Abhängige } \\
\text { Variable }\end{array}$ & Typ III QS & df & MQ & $\mathrm{F}$ & Sig & $\eta_{p}^{2}$ \\
\hline \multirow[t]{9}{*}{ Rumpfneigung } & LUT & 4991,7 & 1,9 & 2683,5 & 54,2 & $<0,001$ & 0,541 \\
\hline & RUT & 4300,6 & 1,4 & 3023,9 & 43,2 & $<0,001$ & 0,485 \\
\hline & LLT & $16,977,5$ & 1,4 & $12,080,1$ & 68,4 & $<0,001$ & 0,598 \\
\hline & RLT & $38,769,8$ & 1,6 & $23,729,5$ & 136,7 & $<0,001$ & 0,748 \\
\hline & LAD & 3902,4 & 1,9 & 2091,7 & 8,4 & 0,001 & 0,154 \\
\hline & RAD & 2537,4 & 1,6 & 1615,8 & 7,0 & 0,004 & 0,132 \\
\hline & LES & $17,125,3$ & 2,0 & 8481,6 & 30,0 & $<0,001$ & 0,395 \\
\hline & RES & $13,591,1$ & 2,1 & 6628,3 & 26,8 & $<0,001$ & 0,368 \\
\hline & RPE & 923,6 & 2,2 & 427,0 & 108,0 & $<0,001$ & 0,701 \\
\hline \multirow[t]{9}{*}{ Schulterflexion } & LUT & $30,422,9$ & 1,6 & $19,613,7$ & 152,1 & $<0,001$ & 0,768 \\
\hline & RUT & $29,293,1$ & 1,4 & $20,470,9$ & 156,8 & $<0,001$ & 0,773 \\
\hline & LLT & $40,747,0$ & 1,4 & $28,325,1$ & 172,8 & $<0,001$ & 0,790 \\
\hline & RLT & $40,233,1$ & 2,0 & $20,125,4$ & 218,3 & $<0,001$ & 0,826 \\
\hline & LAD & $22,846,2$ & 1,6 & $13,914,2$ & 79,8 & $<0,001$ & 0,634 \\
\hline & RAD & $16,873,5$ & 1,6 & $10,246,2$ & 98,8 & $<0,001$ & 0,682 \\
\hline & LES & $29,026,4$ & 1,6 & $17,858,5$ & 172,6 & $<0,001$ & 0,790 \\
\hline & RES & $31,396,6$ & 1,6 & $19,946,6$ & 162,0 & $<0,001$ & 0,779 \\
\hline & RPE & 1080,0 & 2,1 & 513,9 & 336,8 & $<0,001$ & 0,880 \\
\hline \multirow{9}{*}{$\begin{array}{l}\text { Rumpfneigung } \times \\
\text { Schulterflexion }\end{array}$} & LUT & 3856,9 & 3,6 & 1083,4 & 35,5 & $<0,001$ & 0,436 \\
\hline & RUT & 4111,2 & 2,9 & 1416,2 & 38,6 & $<0,001$ & 0,456 \\
\hline & LLT & 6642,6 & 4,0 & 1647,7 & 44,2 & $<0,001$ & 0,490 \\
\hline & RLT & 5323,4 & 3,8 & 1391,9 & 19,1 & $<0,001$ & 0,293 \\
\hline & LAD & 106,6 & 4,5 & 24,0 & 0,7 & 0,585 & 0,016 \\
\hline & RAD & 264,1 & 4,0 & 66,1 & 2,3 & 0,059 & 0,048 \\
\hline & LES & 1196,3 & 5,2 & 229,3 & 11,2 & $<0,001$ & 0,196 \\
\hline & RES & 935,1 & 5,1 & 183,7 & 7,2 & $<0,001$ & 0,135 \\
\hline & RPE & 47,9 & 6,6 & 7,3 & 12,7 & $<0,001$ & 0,216 \\
\hline
\end{tabular}

unabhängigen Variablen auf jede einzelne abhängige Variable zu untersuchen, wurden außerdem separate univariate Varianzanalysen (ANOVA) auf einem Signifikanzniveau von $\alpha=5 \%$ durchgeführt. Sofern gemäß dem Mauchly-Test auf Sphärizität eine Verletzung der Sphärizitätsannahme vorlag, wurde eine Korrektur nach Greenhouse-Geisser durchgeführt.

Die statistische Datenauswertung wurde mit der Software IBM SPSS Statistics 22 durchgeführt.

\section{Ergebnisse}

\subsection{Ergebnisse der Varianzanalyse}

Eine inferenzstatistische Untersuchung der Ergebnisse mittels einer multivariaten Varianzanalyse mit Messwiederholung (MANOVA) und einem Signifikanzniveau von $\alpha=5 \%$ zeigt bei Verwendung der Pillai-Spur einen si- gnifikanten Haupteffekt der Ventralneigung des Rumpfes auf die normierte elektrische Muskelaktivität (sEA) aller Muskeln und das Beanspruchungsempfinden $(\mathrm{V}=1,208$; $\mathrm{F}(27,396)=9,886 ; p<0,001)$. Auch der Haupteffekt der Flexion der Schulter hat einen signifikanten Einfluss auf die sEA aller Muskeln und das Beanspruchungsempfinden $(\mathrm{V}=1,367 ; \mathrm{F}(27,396)=12,276 ; p<0,001)$. Die Interaktion der beiden Faktoren Ventralneigung des Rumpfes und Flexion der Schulter hat einen signifikanten Einfluss auf die sEA aller Muskeln und das Beanspruchungsempfinden $(\mathrm{V}=0,855 ; \mathrm{F}(81,3726)=4,830 ; p<0,001)$.

Ein Einfluss von Muskelermüdung wurde durch eine Randomisierung der Reihenfolge der Faktorstufenkombinationen Ventralneigungswinkel und Schulterflexionswinkel ausgeschlossen. In einer Kovarianzanalyse wurde die Reihenfolge der Faktorstufenkombinationen als Zwischensubjektfaktor untersucht, es ergab sich kein statistisch signifikanter Unterschied zwischen den Bedingungen der 

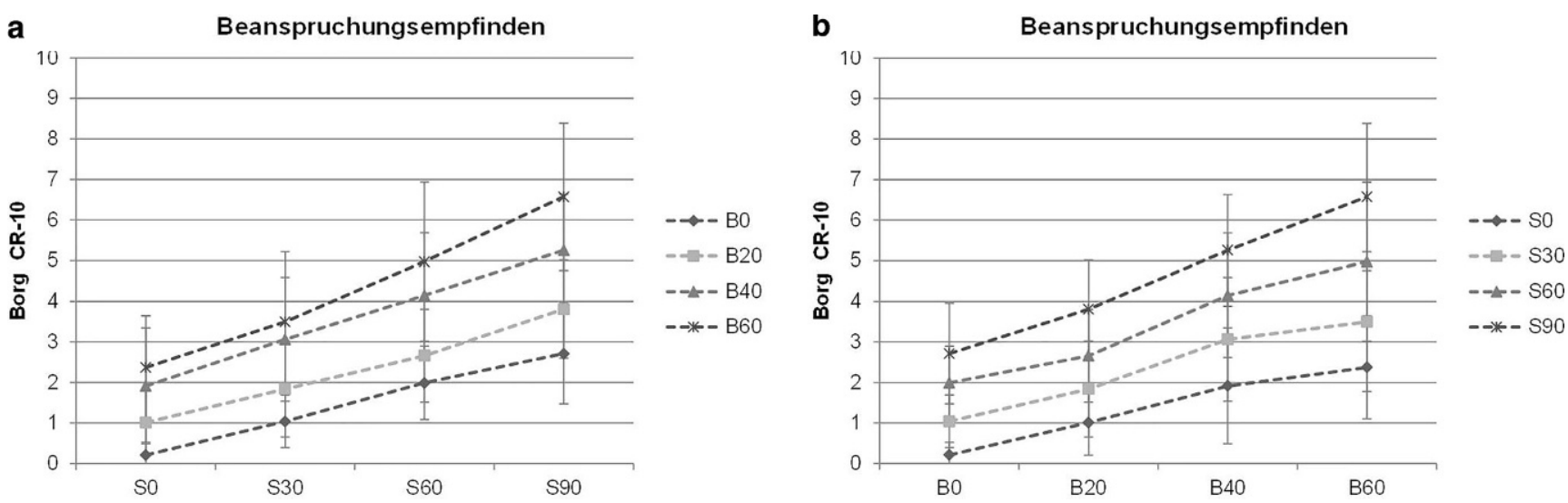

Abb. 2 Interaktionseffekt auf das mittlere Beanspruchungsempfinden. a Schulterflexion $\times$ Rumpfneigung, b Rumpfneigung $\times$ Schulterflexion (B Rumpfneigungswinkel, $S$ Schulterflexionswinkel, Fehlerbalken zeigen Standardabweichung)

Fig. 2 Interaction effect on averaged rating of perceived exertion across subjects according to (a) shoulder flexion $\times$ back inclinaion, (b) back inclination $\times$ shoulder flexion ( $B$ Back inclination angle, $S$ Shoulder inclination angle, error bars indicating standard deviation)

Reihenfolge der Faktorstufenkombinationen $(\mathrm{V}=0,195$; $\mathrm{F}(8,38)=1,148 ; p=0,355)$.

Separate ANOVAs als post-hoc-Tests der MANOVA mit einem Signifikanzniveau von $\alpha=5 \%$ zeigen ebenfalls signifikante Effekte sowohl der Ventralneigung des Rumpfes als auch der Flexion der Schulter auf die sEA der einzelnen Muskeln sowie auf das Beanspruchungsempfinden. Tab. 1 zeigt die Ergebnisse der separaten ANOVAs, bei verletzter Sphärizitätsannahme werden hier nach Greenhouse-Geisser korrigierte Werte angegeben.

Die signifikante Interaktion der beiden Faktoren Ventralneigung des Rumpfes und Flexion der Schulter auf die sEA des Beanspruchungsempfindens und das Beanspruchungsempfinden belegen die wechselseitige Beeinflussung der beiden Faktoren und damit die einleitend beschriebene These, dass eine Beurteilung von Körperhaltung nur unter Berücksichtigung von Wechselwirkungen von Körperteilhaltungen erfolgen darf.

\subsection{Interaktionseffekte}

\subsubsection{Beanspruchungsempfinden (Borg CR-10)}

Die Interaktionseffekte der Ventralneigung des Rumpfes und der Flexion der Schulter haben einen signifikanten Einfluss auf das Beanspruchungsempfinden $(p<0,001)$.

Für alle Ventralneigungswinkel nimmt mit steigendem Schulterflexionswinkel das Beanspruchungsempfinden zu. Bei einem jeweils ähnlichen Verlauf ist das Niveau des Beanspruchungsempfindens insgesamt höher je größer der Ventralneigungswinkel ist (Abb. 2a).

Das Beanspruchungsempfinden nimmt ebenfalls für alle Schulterflexionswinkel mit steigender Ventralneigung des Rumpfes zu. Auch hier nimmt bei vergleichbaren Verläufen das Niveau des Beanspruchungsempfindens mit zunehmendem Flexionswinkel der Schulter ebenfalls zu (Abb. 2b).

\subsubsection{Normierte Muskelaktivität}

Die Interaktionseffekte der Ventralneigung des Rumpfes und der Flexion der Schulter haben einen signifikanten Einfluss auf die sEA des LUT und RUT $(p<0,001)$.

Für alle Rumpfneigungswinkel hat die Haltung der Schulter einen Einfluss auf die sEA (Abb. 3a). Während bei einer Schulterflexion von $0^{\circ}$ die sEA des UT für alle Ventralneigungswinkel des Rumpfes ähnlich ist, entwickelt sich die sEA mit zunehmendem Schulterflexionswinkel stark auseinander.

Die Haltung des Rumpfes hat für größere Schulterflexionswinkel einen stärkeren Einfluss auf die sEA als für kleinere Schulterflexionswinkel (Abb. 3b). Für höhere Schulterflexionswinkel ab $30^{\circ}$ kann mit zunehmender Rumpfneigung ein Anstieg der sEA beobachtet werden. Eine Ausnahme bildet der Verlauf der sEA für eine Schulterflexion von $0^{\circ}$, welcher bei zunehmendem Ventralneigungswinkel leicht abfällt.

Die Interaktionseffekte der Ventralneigung des Rumpfes und der Flexion der Schulter haben einen signifikanten Einfluss auf die sEA des LLT und RLT $(p<0,001)$.

Der Einfluss der Schulterflexion auf die sEA des LT nimmt mit zunehmendem Ventralneigungswinkel des Rumpfes zu (Abb. 4a). Für eine Ventralneigung des Rumpfes von $0^{\circ}$ steigt die sEA des LLT bei Zunahme der Schulterflexion von $30^{\circ}$ auf $90^{\circ}$ lediglich von $9 \%$ auf $11 \%$ MVC beziehungsweise die sEA des RLT von $11 \%$ auf $13 \% \mathrm{MVC}$ an, während für eine Ventralneigung des Rumpfes von $60^{\circ}$ im gleichen Intervall ein Anstieg von $16 \%$ auf $36 \%$ MVC (LLT) beziehungsweise von $26 \%$ auf $43 \%$ MVC (RLT) zu beobachten ist. 
a

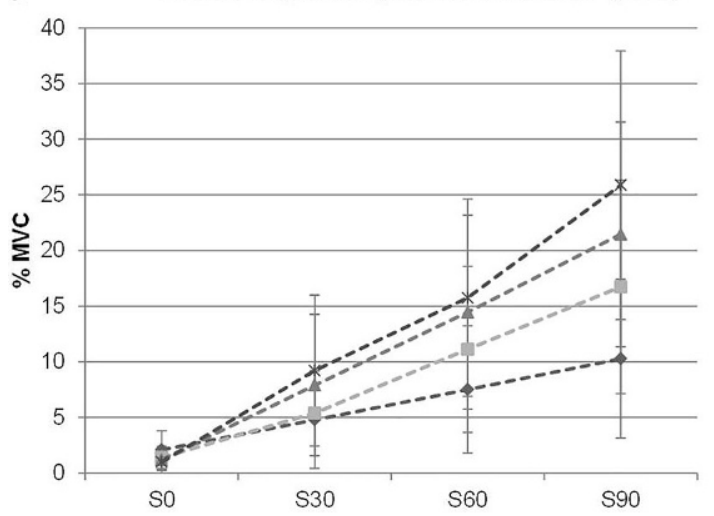

Rechter trapezius pars descendens (RUT)

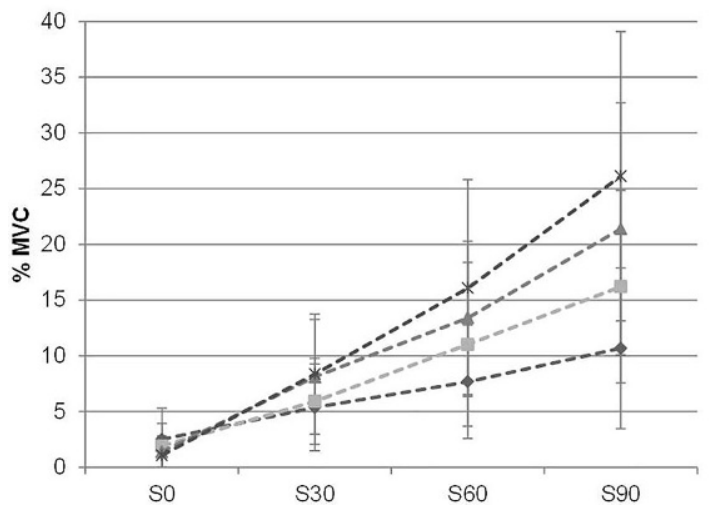

b Linker trapezius pars descendens (LUT)

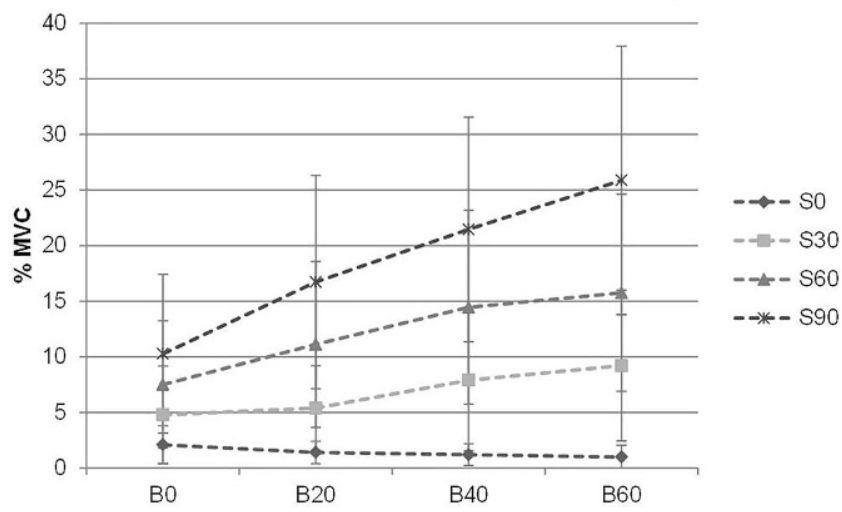

Rechter trapezius pars descendens (RUT)

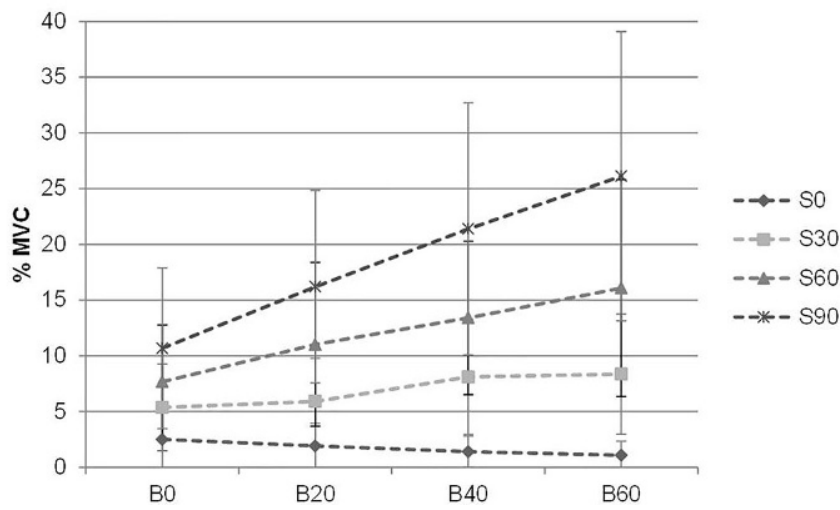

Abb. 3 Interaktionseffekt auf mittlere sEA des UT. a Schulterflexion $\times$ Rumpfneigung. b Rumpfneigung $\times$ Schulterflexion $(B$ Rumpfneigungswinkel, $S$ Schulterflexionswinkel, Fehlerbalken zeigen Standardabweichung)

Fig. 3 Interaction effect on averaged sEA of UT according to (a) shoulder flexion $\times$ back inclination, (b) back inclination $\times$ shoulder flexion ( $B$ Back inclination angle, $S$ Shoulder inclination angle, error bars indicating standard deviation)

Die Haltung des Rumpfes hat für alle Schulterflexionswinkel einen Einfluss auf die sEA des LT (Abb. 4b). Auch hier lässt sich mit ansteigendem Schulterflexionswinkel ein verhältnismäßig stärkerer Anstieg der sEA bei Steigerung der Ventralneigung beobachten.

Die Interaktionseffekte der Ventralneigung des Rumpfes und der Flexion der Schulter haben keinen signifikanten Einfluss auf die sEA des LAD $(p=0,585)$ und des $\operatorname{RAD}(p=0,059)$. Univariate Varianzanalysen zeigen jedoch einen signifikanten Einfluss sowohl der Ventralneigung des Rumpfes (LAD: $p=0,001$; RAD: $p=0,004$ ) als auch der Flexion der Schulter $(p<0,001$; LAD und RAD) auf die sEA des AD.

Für jede Ventralneigung des Rumpfes liegt die jeweils geringste sEA des $\mathrm{AD}$ bei $0^{\circ}$ Schulterflexion und die höchste sEA bei $90^{\circ}$ Schulterflexion (Abb. 5a). Die sEA des AD ist für die Ventralneigungswinkel $20^{\circ}, 40^{\circ}$ und $60^{\circ}$ bei allen Schulterflexionswinkeln gleich groß. Bemerkenswert ist außerdem, dass eine geringe Auslenkung der Schultern aus dem Nullniveau einen vergleichsweise starken Einfluss auf die sEA des AD hat wohingegen ein weiterer Anstieg des
Schulterflexionswinkels einen deutlich geringeren Einfluss auf die sEA des AD ausübt.

Die Interaktionseffekte der Ventralneigung des Rumpfes und der Flexion der Schulter haben einen signifikanten Einfluss auf die sEA des LES und RES $(p<0,001)$.

Die Schulterflexion hat für alle Ventralneigungswinkel einen Einfluss auf den Verlauf der sEA des ES (Abb. 6a). Dabei fällt der Anstieg der sEA steiler aus für Körperhaltungen mit größeren Ventralneigungswinkeln. Für eine Ventralneigung von $60^{\circ}$ verdreifacht sich der Wert der sEA des LES und RES über die gesamte Variationsbreite der Schulterflexion, während sich für eine Ventralneigung von $40^{\circ}$ der Wert der sEA über die gesamte Variationsbreite der Schulterflexion verdoppelt.

Im Hinblick auf den Einfluss der Ventralneigung des Rumpfes ist auffällig, dass für jeden Flexionswinkel die jeweils geringste sEA bei $0^{\circ}$ Ventralneigung vorliegt, ein Anstieg der sEA bis $40^{\circ}$ Ventralneigung auftritt und für eine Ventralneigung von $60^{\circ}$ ein Abfall der sEA zu verzeichnen ist (Abb. 6b). 
a

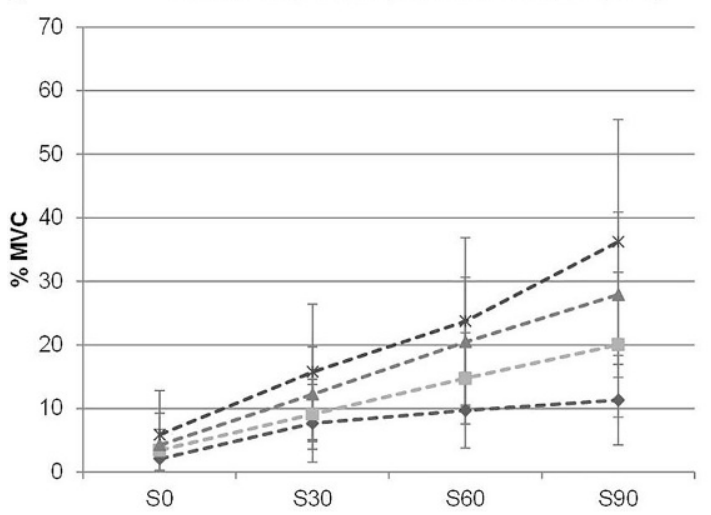

Rechter trapezius pars ascendens (RLT)

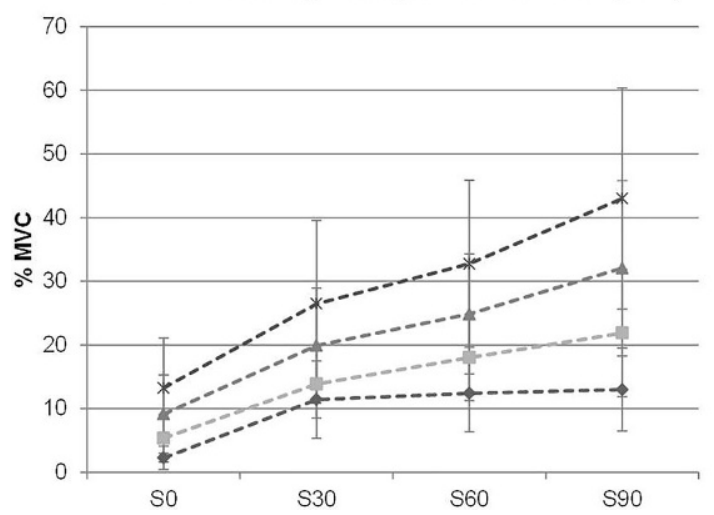

b Linker trapezius pars ascendens (LLT)

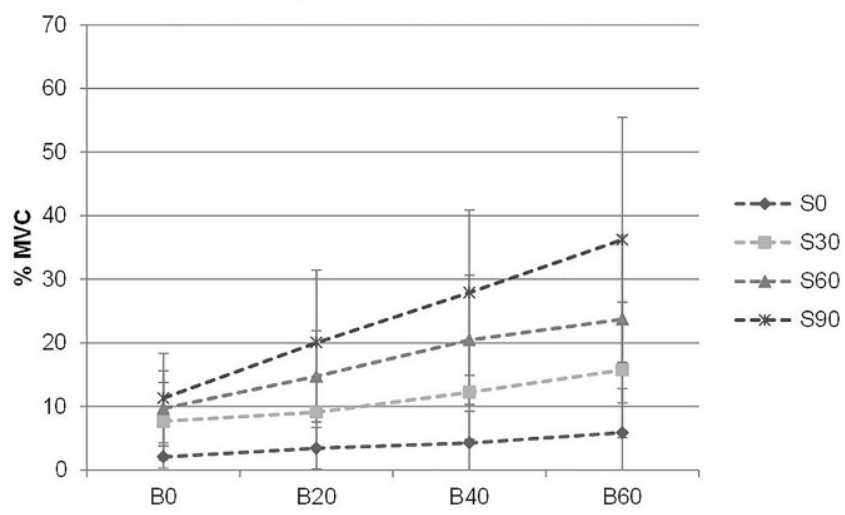

Rechter trapezius pars ascendens (RLT)

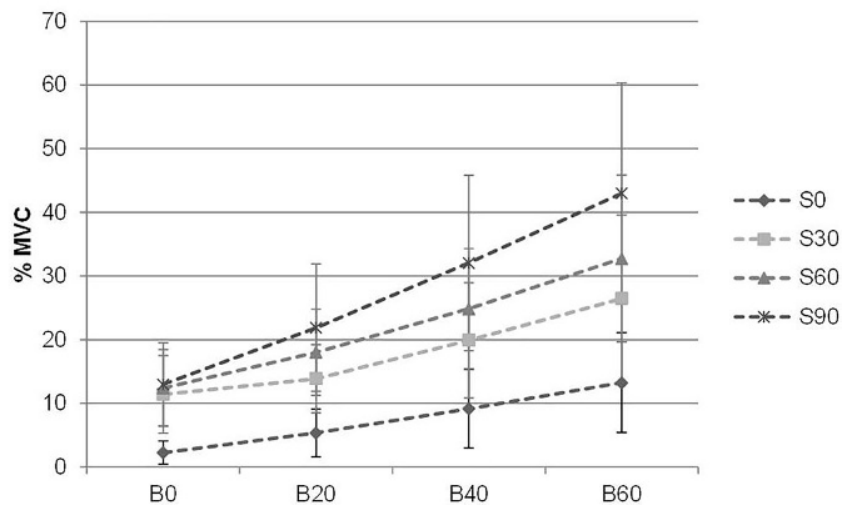

Abb. 4 Interaktionseffekt auf mittlere sEA des LT. a Schulterflexion $\times$ Rumpfneigung. b Rumpfneigung $\times$ Schulterflexion $(B$ Rumpfneigungswinkel, $S$ Schulterflexionswinkel, Fehlerbalken zeigen Standardabweichung)

Fig. 4 Interaction effect on averaged sEA of LT according to (a) shoulder flexion $\times$ back inclination, $(\mathbf{b})$ back inclination $\times$ shoulder flexion $(B$ Back inclination angle, $S$ Shoulder inclination angle, error bars indicating standard deviation)

\section{Diskussion}

Zur Untersuchung der Wechselwirkungen von Körperteilhaltungen des Rückens und der Schulter wurden in dieser Studie die normierte elektrische Muskelaktivität (sEA) von acht Muskeln und das Beanspruchungsempfinden unter systematischer Variation der Ventralneigung des Rumpfes und der Flexion der Schulter erfasst. Die signifikanten Interaktionseffekte der statistischen Datenauswertung belegen die Wechselwirkungen von Körperteilhaltung auf die Beanspruchung des muskuloskelettalen Systems.

\subsection{Beanspruchungsempfinden}

Der Verlauf des Beanspruchungsempfindens ist durch Wechselwirkungen der Ventralneigung des Rumpfes und der Flexion der Schulter geprägt. Lim et al. (2011) konnten durch ihre Untersuchungen ebenfalls Wechselwirkungen der Ventralneigung des Rumpfes und der Flexion der Schulter auf das Beanspruchungsempfinden nachweisen. Das Beanspruchungsempfinden während energetischer Arbeit, in der vorliegenden Studie in Form von Körperhaltung, ist dabei von mehreren Faktoren, wie z.B. der Muskelaktivität aber auch der Muskelermüdung oder der Herzfrequenz abhängig (Pandolf 1978). Die Steigerung des Ventralneigungswinkels des Rumpfes führt in der hier beschriebenen Studie für alle Schulterflexionswinkel zu einem Anstieg des Beanspruchungsempfindens. Unter Berücksichtigung der Studie von Karwowski et al. (1999) sind neben der rein subjektiv erlebten Beanspruchung auch kognitive Prozesse für den Verlauf des Beanspruchungsempfindens verantwortlich. Insbesondere für größere Ventralneigungswinkel und größere Schulterflexionswinkel sowie für deren Kombination kann ein kognitiv bedingter Einfluss auf das Beanspruchungsempfinden nicht ausgeschlossen werden. Eine Beeinflussung des Beanspruchungsempfindens allein durch die Aufgabe, eine Körperhaltung einzunehmen, welche aus den beiden Extremen von Ventralneigungswinkel des Rumpfes und Flexionswinkel der Schulter bestehen, ist denkbar. Dennoch konnten die subjektiv erlebten Zustände der Probanden durch die objektiven Messwerte der sEA bestätigt werden. 
a

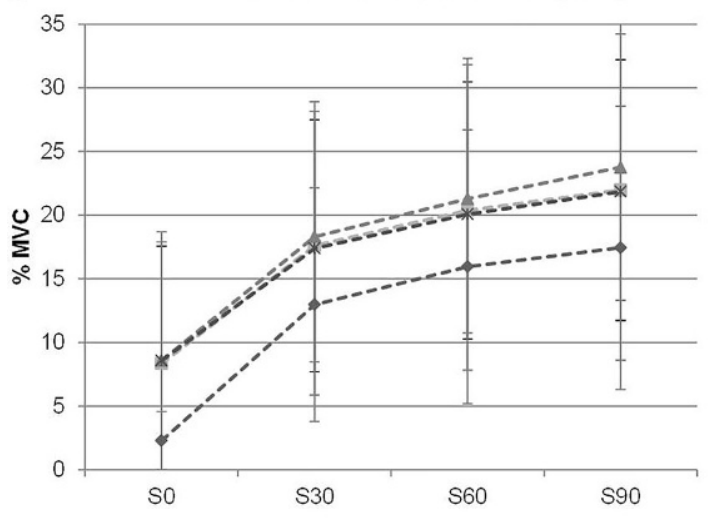

Rechter anterior deltoideus (RAD)

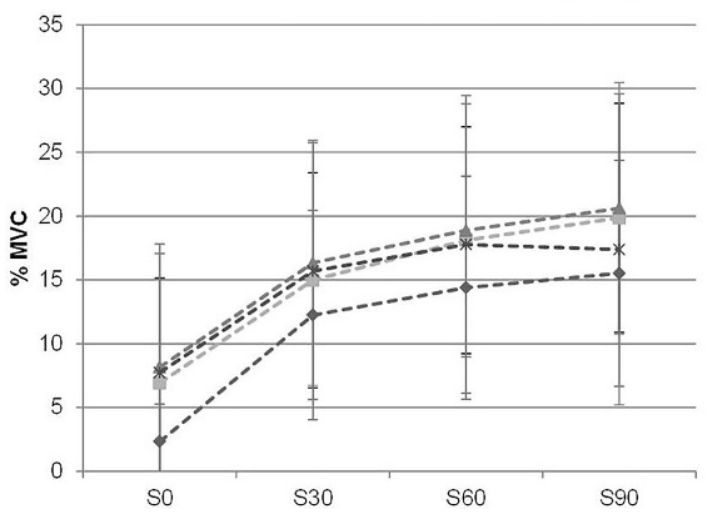

b

Linker anterior deltoideus (LAD)
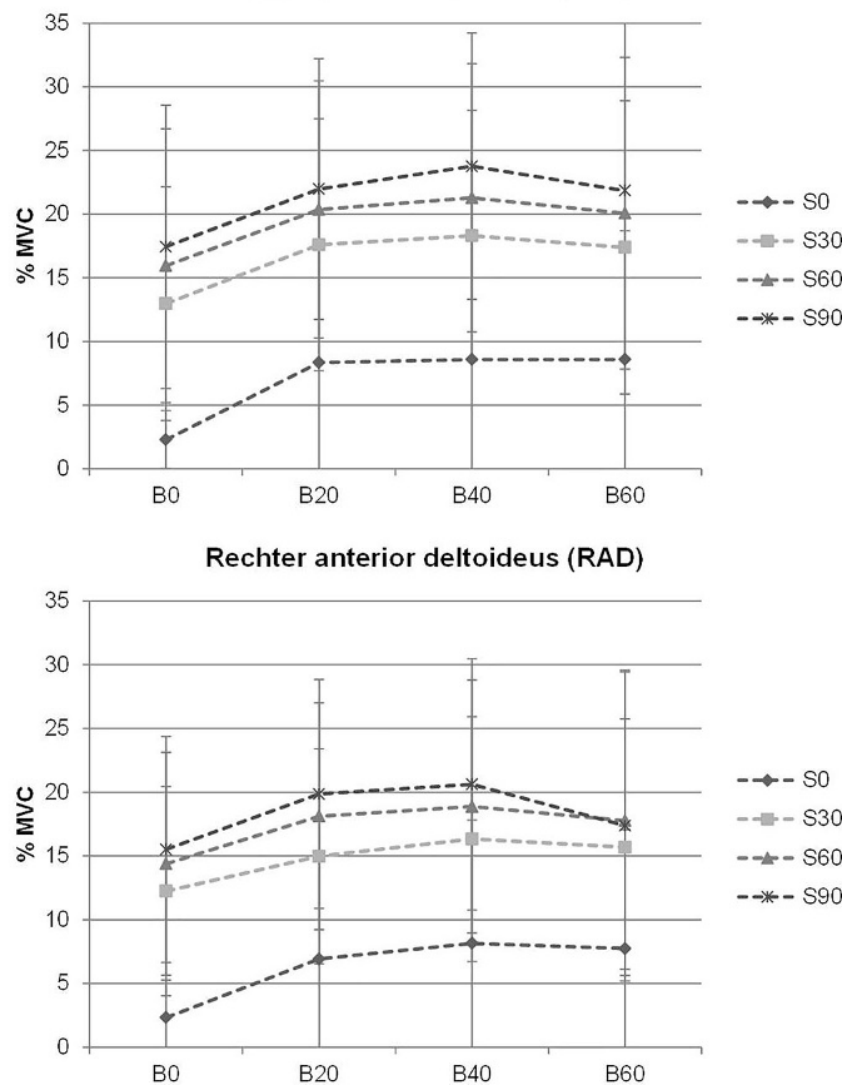

Abb. 5 Interaktionseffekt auf mittlere sEA des AD. a Schulterflexion $\times$ Rumpfneigung. b Rumpfneigung $\times$ Schulterflexion $(B$ Rumpfneigungswinkel, $S$ Schulterflexionswinkel, Fehlerbalken zeigen Standardabweichung)

Fig. 5 Interaction effect on averaged sEA of $\mathrm{AD}$ according to (a) shoulder flexion $\times$ back inclination, (b) back inclination $\times$ shoulder flexion ( $B$ Back inclination angle, $S$ Shoulder inclination angle, error bars indicating standard deviation)

\subsection{Elektrische Muskelaktivität}

Die Funktion des UT ermöglicht das Anheben des Armes durch eine Rotation der Skapula in Zusammenspiel mit dem LT (Perotto und Delagi 2011). Die festgestellte sEA im Ruhezustand bei einer Ventralneigung von $0^{\circ}$ und einer Schulterflexion von $0^{\circ}$ ist mit der notwendigen Grundaktivität des UT zu erklären, welche zur Kompensation des Eigengewichts der oberen Extremität notwendig ist (Schiebler und Korf 2007). Aufgrund einer Verlagerung der Wirkungsrichtung der Gewichtskraft der oberen Extremität mit einem zunehmenden Ventralneigungswinkel, welche auf die Schulter wirkt, ist eine abnehmende sEA des UT bei gleichbleibendem Flexionswinkel der Schulter zu erklären. Lim et al. (2011) konnten ebenfalls bei einem Schulterflexionswinkel von $0^{\circ}$ für einen zunehmenden Ventralneigungswinkel eine abnehmende sEA des UT nachweisen. Der Einfluss der Ventralneigung auf die sEA des UT nimmt mit steigendem Schulterflexionswinkel zu. Diese signifikante Wechselwirkung ist insbesondere bei der Beurteilung der muskuloskelettalen Beanspruchung der oberen Extremitäten zu berücksichtigen. Die Zunahme der sEA des UT mit einem steigenden Schulterflexionswinkel stimmt mit den Ergebnissen der Studie von Kong (2014) überein, welcher die sEA des UT in Abhängigkeit des Schulterflexionswinkels untersuchte und ebenfalls einen Anstieg der sEA mit zunehmendem Schulterflexionswinkel zeigte.

Die Funktion des LT ermöglicht in Zusammenspiel mit dem UT durch eine Rotation der Skapula das Anheben des Armes insbesondere über das Schulterniveau hinweg (Perotto und Delagi 2011). Im Wesentlichen ist der Verlauf der sEA des LT dem Verlauf der sEA des UT sehr ähnlich. Der Einfluss des Ventralneigungswinkels auf die sEA des LT nimmt ebenfalls für steigende Schulterflexionswinkel stark zu. Für einen Ventralneigungswinkel von $60^{\circ}$ nimmt die sEA des LLT um das Sechsfache sowie des RLT um das Vierfache zu und übersteigt im Maximum 35\% MVC. Der Einfluss des Schulterflexionswinkels auf die sEA des LT nimmt für steigende Ventralflexionswinkel ebenfalls stark zu und führt insbesondere für eine Kombination von großen Schulterflexionswinkeln und großen Ventralflexionswinkeln zu hohen Werten der sEA. Auch diese Ergebnisse zeigen, dass neben der Berücksichtigung der Haltung der Schulter für die sEA des LT sowohl die Haltung des 
a
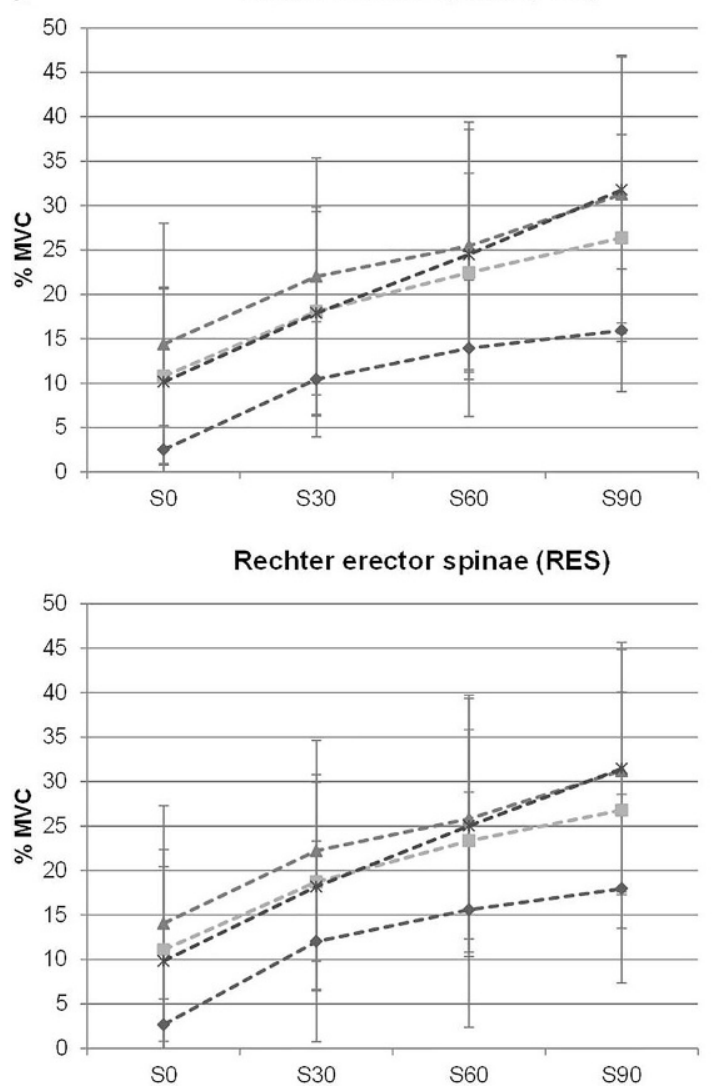

b
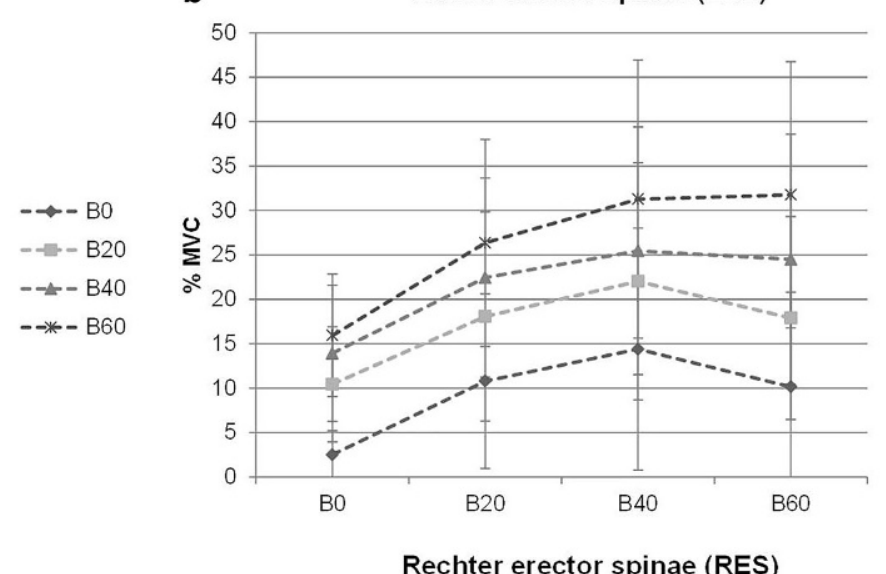

$-\rightarrow-S O$

$--\underline{-1}-\mathrm{S} 30$

$--1-560$

$--*-\$ 90$

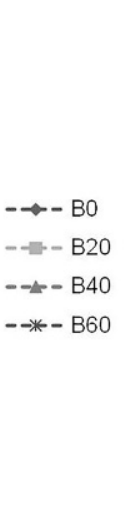

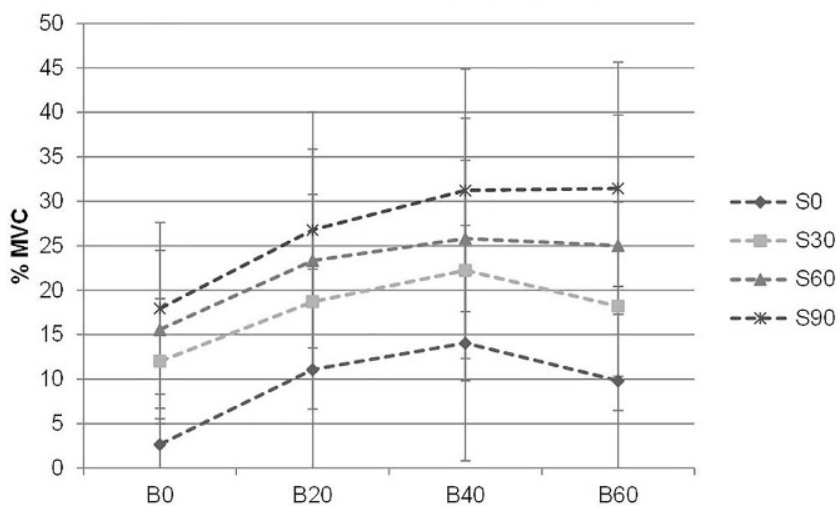

Abb. 6 Interaktionseffekt auf mittlere sEA des ES. a Schulterflexion $\times$ Rumpfneigung. b Rumpfneigung $\times$ Schulterflexion $(B$ Rumpfneigungswinkel, $S$ Schulterflexionswinkel, Fehlerbalken zeigen Standardabweichung)

Fig. 6 Interaction effect on averaged sEA of ES according to (a) shoulder flexion $\times$ back inclination, (b) back inclination $\times$ shoulder flexion $(B$ Back inclination angle, $S$ Shoulder inclination angle, error bars indicating standard deviation)

Rückens als auch Wechselwirkungen zwischen Rücken und Schulter ausschlaggebend sind. Auch diese Ergebnisse sind bei der Beurteilung der muskuloskelettalen Beanspruchung der oberen Extremität zu berücksichtigen.

Für die sEA des AD konnte im Gegensatz zu allen weiteren untersuchten Muskeln keine Wechselwirkungen durch Ventralneigung und Schulterflexion nachgewiesen werden. Dies bedeutet, dass die sEA des AD nicht wechselseitig durch Ventralneigung und Schulterflexion beeinflusst wird. Dennoch üben Ventralneigung und Schulterflexion jeweils signifikante Einflüsse auf die sEA des AD aus. Im Wesentlichen übernimmt der $\mathrm{AD}$ eine unterstützende Funktion von UT und LT bei der Flexion des Armes. Entsprechend den Ergebnissen von Kong (2014) und Lim et al. (2011) ist mit einer steigenden Flexion des Armes ein Anstieg der sEA des AD zu verzeichnen. Für einen Anstieg des Ventralneigungswinkels ist ebenfalls ein Anstieg der sEA des AD zu verzeichnen, jedoch ist bereits bei einem Ventralneigungswinkel von $20^{\circ}$ das Maximum der SEA des AD erreicht. Insofern ist der Einfluss des Schulterflexionswinkels auf die sEA des AD als wesentlicher Faktor anzusehen.
Die Funktion des ES ermöglicht ein Aufrichten des Rumpfes gegen die Schwerkraft durch die Verkürzung des ES während der Muskelkontraktion. Demnach erfolgt die Ausführung einer Ventralflexion des Rumpfes, indem der Rumpf durch die Schwerkraft abgesenkt wird und der ES hierbei gleichzeitig eine bremsende Funktion übernimmt (Schiebler und Korf 2007). Die Wechselwirkung von Ventralneigung und Schulterflexion auf die sEA des ES steht in Übereinstimmung mit den Ergebnissen von Lim et al. (2011). Bei einem angenommenen Drehpunkt des Rumpfes im Bereich des L5-S1 der Wirbelsäule ist der Effekt einer steigenden sEA des ES mit zunehmendem Schulterflexionswinkel durch den vergrößerten Hebelarm des Moments auf den L5-S1 zu erklären. Dieses Moment wird durch die Kontraktion des ES ausgeglichen, um den Rumpf in einer statischen Haltung zu fixieren. Ebenfalls ist der Anstieg der sEA des ES mit zunehmendem Ventralneigungswinkel des Rumpfes auf bis zu $40^{\circ}$ durch ein vergrößertes Moment auf den Drehpunkt des Rumpfes am L5-S1 zu erklären. Die Abnahme der sEA des ES mit einer weiteren Zunahme des Ventralneigungswinkels von $40^{\circ}$ auf $60^{\circ}$ steht in Übereinstimmung mit den Ergebnissen von Jäger 
und Luttmann (1989), welche einen ähnlichen Verlauf der Druckbelastung der Bandscheibe des L5-S1 in Abhängigkeit der Ventralneigung des Rumpfes beschreiben. Als entscheidende Faktoren für diesen Effekt können sowohl der abdominale Druck des Bauchraumes als auch die Entwicklung des statischen Moments auf den Bezugspunkt L5S1 in Abhängigkeit des wirkenden Hebelarms angesehen werden. Zum einen steigt der abdominale Druck des Bauchraumes mit steigender Einengung der Bauchhöhle, welche durch eine zunehmende Ventralneigung verursacht wird. Dieser abdominale Druck kann zu einem kleinen Anteil das wirkende Moment auf den L5-S1 kompensieren (Jäger 1987) und führt damit auch zu einer Abnahme der sEA des ES für sehr große Ventralneigungswinkel. Zum anderen ist die Lage des Bezugspunktes des Moments am L5-S1 eine weitere Ursache für eine abnehmende sEA des ES. Der Bezugspunkt des Moments liegt im Rahmen der biomechanischen Betrachtung relativ zur Längsachse der Wirbelsäule dorsal versetzt im Schwerpunkt der Bandscheibe des L5S1 und nicht am eigentlichen Drehpunkt der Wirbelsäule (Jäger und Luttmann 1989). Bei der Betrachtung der elektrischen Muskelaktivität ist ebenfalls ein dorsaler Versatz des Bezugspunktes des Moments ursächlich für den Abfall der sEA des ES für große Ventralneigungswinkel, da für die Lage des Bezugspunktes des Moments hier die Lage des ES entscheidend ist, welcher gegenüber dem Schwerpunkt der Bandscheibe noch weiter dorsal liegt.

Die im Rahmen dieser Studie erhobenen Verläufe der sEA stimmen im Wesentlichen für die linke und rechte Körperhälfte überein. Tendenziell ist die sEA der linken Muskeln etwas höher als die der rechten Muskeln. Dies spricht einerseits für eine gute Signalqualität und andererseits für einen höheren neuromuskulären Trainingsstatus der Muskeln der rechten Körperhälfte, was auf eine überwiegende Rechtshändigkeit des Kollektivs der Versuchspersonen zurückzuführen ist.

Insgesamt ist festzustellen, dass bereits die Kombination von kleineren Ventralneigungswinkeln und kleineren Schulterflexionswinkeln zu einer teilweise deutlichen $\mathrm{Zu}$ nahme der sEA in den untersuchten Muskeln führt. Bereits Rohmert (1962) nennt eine sEA von mehr als $15 \%$ MVC als Grenzwert, dessen Übersteigung eine Kontraktion der kleinen Blutgefäße der Muskeln bedingt und somit eine Unterversorgung der Muskeln mit Sauerstoff durch eine Unterbindung des Blutflusses verursacht. Weitere Studien belegen zudem einen Zusammenhang von hoher sEA und der Entstehung von Muskel- und Skeletterkrankungen (Ostensvik et al. 2009; Ferguson et al. 2012). Auf Basis der Ergebnisse ist eine Berücksichtigung von Wechselwirkungen von Körperteilhaltungen gerade im Bereich des Rückens und der Schulter bei der Belastungsbewertung von Körperhaltungen dringend geboten. Wie auch Lim et al. (2011) anmerkten, bergen Belastungsbewertungsverfahren i. A. die
Gefahr der Unterbewertung von Risiken durch Körperhaltungen, da diese Verfahren Wechselwirkungen von Körperteilhaltungen üblicherweise nicht berücksichtigen. Insbesondere jedoch die ergonomische Gestaltung von manuellen Arbeitssystemen kann von einer Berücksichtigung der Wechselwirkungen von Körperteilhaltungen profitieren, indem das Risiko des Auftretens von Muskel- und Skeletterkrankungen dadurch weiter gesenkt wird.

\subsection{Limitationen}

Als Limitation der Studie ist zu berücksichtigen, dass diese unter kontrollierten Laborbedingungen durchgeführt wurde und daher eine Generalisierbarkeit der Ergebnisse nur unter Einschränkungen möglich ist. Hinsichtlich der Auswahl der untersuchten Muskeln im Bereich der Schulter ist anzumerken, dass die Schulterflexion eine Bewegung darstellt, welche unter der Beteiligung einer Vielzahl an Muskeln stattfindet. Hierzu gehören neben den untersuchten Muskeln des UT, LT und AD ferner die M. infraspinatus und supraspinatus (Iridiastadi et al. 2008). Obwohl im Rahmen dieser Studie bereits drei Muskeln der Schulterregion untersucht wurden, kann damit nicht das gesamte System zur Ausführung einer Schulterflexion wiedergegeben werden. Ferner wurden im Rahmen dieser Studie keine dynamischen Muskelkontraktionen unter Bewegungsausführung untersucht. Der Transfer der hier dargestellten Ergebnisse beschränkt sich daher auch auf die Analyse und Bewertung von statischen Körperhaltungen. Da jedoch unter der Vielzahl an Belastungsbewertungsverfahren auch solche zur Verfügung stehen, welche sich für eine Untersuchung statischer Körperhaltungen eignen, wie z.B. die statischen Teile der DIN EN 1005-4 (2009) oder auch das OWAS, ist bereits auf Basis der hier vorgestellten Studienergebnisse eine Diskussion der Sensitivität dieser Methoden hinsichtlich der Beurteilung von Wechselwirkungen von Körperteilhaltungen möglich. Ferner ist auch eine Diskussion der Anzahl an Studienteilnehmern notwendig, welche zur Untersuchung des Einflusses von 16 verschiedenen Faktorstufenkombinationen auf neun unabhängige Variablen als relativ klein anzusehen ist. Dennoch konnten im Rahmen dieser Studie statistisch signifikante Interaktionseffekte der untersuchten Faktoren auf subjektive und objektive Beanspruchungsparameter nachgewiesen werden. Zudem sind Diskrepanzen zwischen dem in dieser Studie untersuchten Kollektiv der Studienteilnehmer und geübten Arbeitspersonen industrieller Arbeitssysteme möglich. Um diese Diskrepanzen möglichst klein zu halten, wurden ausschließlich gesunde und in guter körperlicher Verfassung stehende Personen in die Studie eingeschlossen.

Schließlich ist auf Basis der hier beschriebenen Studienergebnisse die Hypothese zu formulieren, dass zwischen weiteren Körperteilen Wechselwirkungen auf die Beanspru- 
chung bestehen. Im Rahmen dieser Studie konnten lediglich Wechselwirkungen der Ventralneigung des Rückens und der Flexion der Schulter auf die sEA und das Beanspruchungsempfinden untersucht werden.

\section{Schlussfolgerung}

Die im Rahmen dieser Studie dargestellten Ergebnisse stellen einen ersten Schritt zur Berücksichtigung von Wechselwirkungen von Körperteilhaltungen bei der Belastungsbewertung dar. Signifikante Interaktionseffekte zwischen der Ventralneigung des Rumpfes und der Schulterflexion konnten auf Basis empirischen Datenmaterials statistisch nachgewiesen werden. Dies zeigt, dass eine Berücksichtigung von Wechselwirkungen von Körperteilhaltungen zur Steigerung der Validität der Ergebnisse von Belastungsbewertungen von essentieller Bedeutung ist. Eine Belastungsbewertung, welche auf der isolierten Betrachtung von einzelnen Körperteilen beruht, wird zu fehlerhaften Bewertungsergebnissen führen. Diese Einschätzung gibt neben Lim et al. (2011) ebenfalls die DIN EN 1005-4 (2009) wieder. Aufgrund der Komplexität von statistischen Interaktionseffekten besteht die Notwendigkeit, die Anwendung dieser Erkenntnisse durch praxistaugliche Methoden zu ermöglichen. Dies ist bspw. durch die Anwendung von Sensoren und Motion Capture Systemen im Bereich der ergonomischen Analyse und Intervention denkbar (Brandl et al. 2017a). Der Prozess der Belastungsbewertung kann durch eine sensorgestützte Erfassung sowie durch Algorithmen gestützte Bewertung von Körperhaltung deutlich vereinfacht werden. Um Muskel- und Skeletterkrankungen in Zukunft wirksamer begegnen zu können, ist eine Berücksichtigung von Wechselwirkungen bei der Bewertung von Körperhaltungen unbedingt zu empfehlen.

Förderung Das diesem Beitrag zugrundeliegende Forschungsvorhaben „ENgAge4Pro“ wurde mit Mitteln des BMBF (FKZ: 16SV6143) gefördert. Projektträger war der VDI/VDE-IT. Die Open Access Gebühr dieses Beitrags wurde mit Mitteln des BMBF (FKZ: 16PGF0109) gefördert.

Open Access Dieser Artikel wird unter der Creative Commons Namensnennung 4.0 International Lizenz (http://creativecommons.org/ licenses/by/4.0/deed.de) veröffentlicht, welche die Nutzung, Vervielfältigung, Bearbeitung, Verbreitung und Wiedergabe in jeglichem Medium und Format erlaubt, sofern Sie den/die ursprünglichen Autor(en) und die Quelle ordnungsgemäß nennen, einen Link zur Creative Commons Lizenz beifügen und angeben, ob Änderungen vorgenommen wurden.

\section{Literatur}

Borg G (1990) Psychophysical scaling with applications in physical work and the perception of exertion. Scand J Work Environ Health 16(Suppl 1):55-58
Brandl C (2017) Ergonomische Analyse von Körperhaltungen in Produktionssystemen für eine computergestützte Arbeitsgestaltung und -organisation. RWTH Aachen, Aachen (Dissertation)

Brandl C, Bonin D, Mertens A, Wischniewski S, Schlick CM (2016) Digitalisierungsansätze ergonomischer Analysen und Interventionen am Beispiel der markerlosen Erfassung von Körperhaltungen bei Arbeitstätigkeiten in der Produktion. Z Arb Wiss 70(2):89-98. https://doi.org/10.1007/s41449-016-0016-9

Brandl C, Mertens A, Schlick CM (2017a) Effect of sampling interval on the reliability of ergonomic analysis using the Ovako working posture analysing system (OWAS). Int J Ind Ergon 57:68-73. https://doi.org/10.1016/j.ergon.2016.11.013

Brandl C, Mertens A, Schlick CM (2017b) Ergonomic analysis of working postures using OWAS in semi-trailer assembly, applying an individual sampling strategy. Int J Occup Saf Ergon 23(1):110-117. https://doi.org/10.1080/10803548.2016.1191224

David GC (2005) Ergonomic methods for assessing exposure to risk factors for work-related musculoskeletal disorders. Occup Med (Chic Ill) 55(3):190-199. https://doi.org/10.1093/occmed/kqi082

Denis D, Lortie M, Rossignol M (2000) Observation procedures characterizing occupational physical activities. Critical review. Int J Occup Saf Ergon 6(4):463-491. https://doi.org/10.1080/ 10803548.2000.11076467

DIN EN 1005-4, 2009-01: Sicherheit von Maschinen - Menschliche körperliche Leistung - Teil 4: Bewertung von Körperhaltungen und Bewegungen bei der Arbeit an Maschinen.

Farooq M, Khan AA (2014) Effects of shoulder rotation combined with elbow flexion on discomfort and EMG activity of ECRB muscle. Int J Ind Ergon 44(6):882-891. https://doi.org/10.1016/ j.ergon.2013.10.010

Ferguson SA, Marras WS, Allread WG, Knapik GG, Splittstoesser RE (2012) Musculoskeletal disorder risk during automotive assembly. Current vs. seated. Appl Ergon 43(4):671-678. https://doi.org/10. 1016/j.apergo.2011.10.001

Field A (2013) Discovering statistics using IBM SPSS statistics, 4. Aufl. SAGE, Los Angeles, London, New Delhi, Singapore, Washington DC

Hermens HJ (1999) European Recommendations for Surface ElectroMyoGraphy // European recommendations for surface ElectroMyoGraphy. Results of the SENIAM project. Roessingh Research and Development, Enschede (SENIAM, 8)

Iridiastadi H, Nussbaum MA (2006) Muscle fatigue and endurance during repetitive intermittent static efforts. Development of prediction models. Ergonomics 49(4):344-360. https://doi.org/10. 1080/00140130500475666

Iridiastadi H, Nussbaum MA, van Dieën JH (2008) Muscular load characterization during isometric shoulder abductions with varying force. J Electromyogr Kinesiol 18(4):695-703. https://doi.org/ 10.1016/j.jelekin.2007.01.011

Jäger M (1987) Biomechanisches Modell des Menschen zur Analyse und Beurteilung der Belastung der Wirbelsäule bei der Handhabung von Lasten. Universität, Dortmund (Dissertation)

Jäger M, Luttmann A (1989) Biomechanical analysis and assessment of lumbar stress during load lifting using a dynamic 19-segment human model. Ergonomics 32(1):93-112. https://doi.org/ $10.1080 / 00140138908966070$

Karhu O, Härkönen R, Sorvali P, Vepsäläinen P (1981) Observing workin postures in industry: examples of OWAS application. Appl Ergon 12(1):13-17. https://doi.org/10.1016/0003-6870(81)90088-0

Karwowski W, Lee W, Jamaldin B, Gaddie P, Jang RL, Alqesaimi KK (1999) Beyond psychophysics. The need for a cognitive engineering approach to setting limits in manual lifting tasks. Ergonomics 42(1):40-60. https://doi.org/10.1080/001401399185793

Khan AA, O'Sullivan L, Gallwey TJ (2009) Effects of combined wrist flexion/extension and forearm rotation and two levels of relative force on discomfort. Ergonomics 52(10):1265-1275. https://doi. org/10.1080/00140130903040208 
Kluth K, Steinhilber B, Nesseler T (2013) Oberflächen-Elektromyographie in der Arbeitsmedizin, Arbeitsphysiologie und Arbeitswissenschaft. Z Arb Wiss 67(2):113-128. https://doi.org/10.1007/ BF03373911

Kong Y-K (2014) The effects of co-ordinating postures with shoulder and elbow flexion angles on maximum grip strength and upperlimb muscle activity in standing and sitting postures. Int $\mathrm{J}$ Occup Saf Ergon 20(4):595-606. https://doi.org/10.1080/10803548. 2014.11077077

Lim C-M, Myung-Chul J, Kong Y-K (2011) Evaluation of upper-limb body postures based on the effects of back and shoulder flexion angles on subjective discomfort ratings, heart rates and muscle activities. Ergonomics 54(9):849-857. https://doi.org/10.1080/ 00140139.2011.600777

Luger T, Bosch T, Hoozemans M, de Looze M, Veeger D (2015) Task variation during simulated, repetitive, low-intensity work-influence on manifestation of shoulder muscle fatigue, perceived discomfort and upper-body postures. Ergonomics 58(11):18511867. https://doi.org/10.1080/00140139.2015.1043356

McAtamney L, Corlett NE (1993) RULA: a survey method for the investigation of work-related upper limb disorders. Appl Ergon 24(2):91-99. https://doi.org/10.1016/0003-6870(93)90080-S

Ostensvik T, Veiersted KB, Nilsen P (2009) Association between numbers of long periods with sustained low-level trapezius muscle activity and neck pain. Ergonomics 52(12):1556-1567. https://doi. org/10.1080/00140130903199889

Pandolf KB (1978) Influence of local and central factors in dominating rated perceived exertion during physical work. Percept Mot Skills 46(3 Pt 1):683-698. https://doi.org/10.2466/pms.1978.46.3.683

Perotto A, Delagi EF (2011) Anatomical guide for the electromyographer. The limbs and trunk, 5. Aufl. Charles C. Thomas, Springfield

Plewa K, Potvin JR, Dickey JP (2016) Wrist rotations about one or two axes affect maximum wrist strength. Appl Ergon $53 \mathrm{Pt}$ A:152-160. https://doi.org/10.1016/j.apergo.2015.09.005

Robert Koch-Institut (2015) Bericht Gesundheit in Deutschland 2015 (zuletzt geprüft am 01.08.2017)
Rohmert W (1962) Untersuchungen über Muskelermüdung und Arbeitsgestaltung. RWTH Aachen, Aachen (Habilitationsschrift)

Rohmert W (1983) Formen menschlicher Arbeit. In: Rohmert W, Rutenfranz J (Hrsg) Praktische Arbeitsphysiologie, 3. Aufl. Thieme, Stuttgart, New York, S 5-29

Roman-Liu D (2014) Comparison of concepts in easy-to-use methods for MSD risk assessment. Appl Ergon 45(3):420-427. https://doi. org/10.1016/j.apergo.2013.05.010

Schiebler TH, Korf H-W (2007) Anatomie. Histologie, Entwicklungsgeschichte, makroskopische und mikroskopische Anatomie, Topographie, 10. Aufl. Steinkopff, Darmstadt

Schlick CM, Luczak H, Bruder R (2018) Arbeitswissenschaft. Springer, Berlin, Heidelberg

Sousa A, Tavares J (2012) Surface electromyographic amplitude normalization methods. A review. In: Electromyography: new developments, procedures and applications

Srinivasan D, Sinden KE, Mathiassen SE, Côté JN (2016) Gender differences in fatigability and muscle activity responses to a shortcycle repetitive task. Eur J Appl Physiol 116(11-12):2357-2365. https://doi.org/10.1007/s00421-016-3487-7

Takala E-P, Pehkonen I, Forsman M, Hansson G-Å, Mathiassen SE, Erik S, Neumann WP et al (2010) Systematic evaluation of observational methods assessing biomechanical exposures at work. Scand J Work Environ Health 36(1):3-24. https://doi.org/10. 5271/sjweh.2876

Widanarko B, Legg S, Stevenson M, Devereux J, Eng A, Mannetje A et al (2011) Prevalence of musculoskeletal symptoms in relation to gender, age, and occupational/industrial group. Int J Ind Ergon 41(5):561-572. https://doi.org/10.1016/j.ergon.2011.06.002

Widanarko B, Legg S, Stevenson M, Devereux J, Eng A, Mannetje A et al (2012) Gender differences in work-related risk factors associated with low back symptoms. Ergonomics 55(3):327-342. https:// doi.org/10.1080/00140139.2011.642410 